\title{
EDITORIAL
}

\section{Competencias prácticas en la Anatomía humana, tarea difícil durante la pandemia por coronavirus.}

\section{Practical competences in Human Anatomy, a difficult task during the coronavirus pandemic.}

Franklin Miranda-Solís. MD. ${ }^{1}$, Jaquelin Montes-Navarro. Bch. ${ }^{2}$

$\mathrm{D}$ esde que se declara el estado de emergencia en el estado peruano, el 15 marzo del 2020. Los laboratorios de anatomía humana de las diferentes universidades del Perú paralizan sus actividades, esto pone como reto, poder adaptarse a las herramientas de conectividad y tratar de continuar con la labor de seguir enseñando. Pero por su naturaleza, el curso de anatomía tiene un componente netamente practico, y son pocas las herramientas virtuales con las que se cuentan para la enseñanza, pero estos no remplazan la experiencia de poder ver y explorar en el laboratorio a un espécimen cadavérico, y contribuir sustancialmente en el aprendizaje de la anatomía ligada al desarrollo de habilidades manuales obtenidas con la disección cadavérica.

Por otra parte se sabe que los resultados de aprendizaje dependen en gran medida de los conocimientos(1), habilidades y valores adquiridos o desarrollados previamente por los estudiantes, sin embargo, es también importante la complementación del nivel de motivación que pueda impartirse desde las aulas, y en ello juega un papel fundamental los laboratorios donde se haga uso de material cadavérico, en ese sentido la pandemia por el coronavirus ha detenido esta parte de la experiencia y motivación en los laboratorios envista a las restricciones por el estado de emergencia.

Además, se conoce que existe una positiva correlación entre la disección y el rendimiento en anatomía (2-6), por lo que retornar a los laboratorios de anatomía se hace una necesidad. Por otra parte, la pandemia por la Covid-19 ha creado una crisis a nivel mundial ocasionando un severo impacto en la formación médica, no obstante, la educación virtual es el modo actual de enseñanza en el escenario actual (7), sin embargo, la comprensión de los contenidos sin disección u otras ayudas prácticas de enseñanza como huesos, especímenes, etc. es un reto. La posible perturbación educativa se siente actualmente y se experimentará inclusive después que haya terminado la pandemia debido a los escases de cadáveres que se experimentará más adelante, esto conllevará a que los cadáveres reales serán reemplazados por cadáveres virtuales debido a la disminución o poca donación de cadáveres o restricción que se tengan por riesgo a la contaminación por el coronavirus. Así mismo la investigación en el campo de la anatomía también se verá afectada negativamente (8).

Un aspecto que impactó negativamente durante el confinamiento es la disminución o nula participación de los estudiantes, esto en función a la implementación brusca del aprendizaje a distancia ya sea sincrónica o asincrónica (9), produciendo mayor riesgo de ansiedad a lo ocasionado por la misma crisis de la Covid-19(10). la población estudiantil no es ajena a ello pues los estudiantes refieren a una disminución en la calidad de vida, y el estrés afecta negativamente en la motivación de estos, así como en el desempeño académico $(11,12)$. Por lo tanto, el impacto de la restricción al desarrollo de las actividades prácticas en los laboratorios de anatomía podría tener consecuencias psicológicas en los estudiantes.

Por los aspectos mencionados, es importante proponer estrategias de acondicionamiento a fututo para el retorno a los laboratorios, así como implementar los procesos de donación de cuerpos, con el propósito de disminuir los riesgos de transmisión del virus SARS-CoV-2 en los ambientes de práctica.

\section{REFERENCIAS BIBLIOGRAFICAS}

1. Carrillo M, Padilla J, Rosero T, Sol Villagómez M. La motivación y el aprendizaje. Alteridad. 2009;4(1):20-33.

2. Lachman N, Pawlina W. Integrating professionalism in early medical education: The theory and application of reflective practice in the anatomy curriculum. Clinical Anatomy. 2006;19(5):456-60.

3. Nwachukwu C, Lachman N, Pawlina W. Evaluating dissection in the gross anatomy course: Correlation between quality of laboratory dissection and station between qualiy of lass outcomes. Anatomical Sciences Education. 2015;8(1):45-
52 .

4. Rae G, Cork RJ, Karpinski AC, Swartz WJ. The integration of brain dissection within the medical neuroscience laboratory enhances learning. Anatomical Sciences Education. 2016;9(6):565-74.
Ramsey-Stewart G, Burgess AW, Hill DA. Back to the future: teaching anatomy by whole-body dissection. Medical Journal of Australia. 2010;193(11-12):668-71.

6. Vorstenbosch MATM, Kooloos JGM, Bolhuis SM, Laan RFJM. An investigation of anatomical competence in
junior medical doctors. Anatomical Sciences Education. junior medical dock;

Pather N, Blyth P, Chapman JA, Dayal MR, Flack NAMS, Fogg QA, et al. Forced Disruption of Anatomy Education in Australia and New Zealand: An Acute Response to the Covid-19 Pandemic. Anatomical Sciences Education. 2020;13(3):284-300.

Singal A, Bansal A, Chaudhary P. Cadaverless anatomy: Darkness in the times of pandemic Covid-19. Morphologie. 2020;104(346):147-50.
9. Longhurst GJ, Stone DM, Dulohery K, Scully D, Campbell T, Smith CF. Strength, Weakness, Opportunity, Threat (SWOT) Analysis of the Adaptations to Anatomical Education in the United Kingdom and Republic of Ireland in Response to the Covid-19 Pandemic. Anatomical Sciences Education. 2020;13(3):301-11.

10. Rubin GJ, Wessely S. The psychological effects of quarantining a city. BMJ. 2020;368:m313.

11. Artino AR, La Rochelle JS, Durning SJ. Second-year medical students' motivational beliefs, emotions, and achievement. Medical Education. 2010;44(12):1203-12.

12. Lyndon MP, Henning MA, Alyami H, Krishna S, Zeng I, Yu TC, et al. Burnout, quality of life, motivation, and academic achievement among medical students: A person-oriented approach. Perspect Med Educ. A person-oriented approach. Perspect Med Educ.

1. Universidad Nacional de San Antonio Abad del Cusco, Lab de Anatomía Humana, Escuela de Medicina Humana, Cusco, Perú

2. Universidad Autónoma de Ica, Escuela profesional de Psicología Humana, Ica, Perú

Citar como : Miranda-Solis F, Montes-Navarro J. Competencias prácticas en la Anatomía humana, tarea difícil durante la pandemia por coronavirus. Rev Peruana de Morfologia. 2020; 1(1):3

Conflicto de interes: FMS declara ser editor de la revista, JMN declara no tener ningun conflicto de interes

Contribucion: FMS, JMN, plantearon el tema, redactaron, aprobaron el documento final.

Recibido: 19/05/2020; Aceptado: 12/08/2020

Autor corresponsal: Franklin Miranda-Solis

mdfranklinm@gmail.com 